\title{
The pathophysiology of cataract and major interventions to retarding its progression: a mini review
}

\begin{abstract}
Cataracts are the principal cause of blindness, globally, affecting the older cohort (specifically those in their fifth decades and beyond). In fact, there are more cases of cataracts worldwide than there are of glaucoma, macular degeneration and diabetic retinopathy (DR) combined, according to Prevent Blindness America (PBA). Whilst ageing remains the predominant risk factor for cataract formation, other predisposing factors such as diabetes mellitus (DM), nutritional and trace element deficiency, ultraviolet radiations, smoking, etc., have been implicated in this sight threatening condition. The pathophysiology of cataract is not fully understood albeit aldose-reductase inhibitors and antioxidants have proven beneficial in the prevention and management of this vision threatening condition in vitro and in vivo experimental studies. This paper seeks to provide an overview of the understanding of the pathophysiology of cataract and the major interventions that have been deployed to help retard its progression, as highlighted in extant literature.
\end{abstract}

Keywords: pathophysiology, cataract, interventions, retarding, progression
Volume 6 Issue 3 - 2017

\author{
Andrews Nartey \\ Department of Optometry and Visual Science, Kwame \\ Nkrumah University of Science and Technology, Ghana \\ Correspondence: Andrews Nartey, Department of \\ Optometry and Visual Science, Kwame Nkrumah University of \\ Science and Technology, Kumasi, Ghana, Tel +233 50 I37 4I48, \\ Email andy8nartey@gmail.com
}

Received: January 04, 2017 | Published: February 22, 2017
Abbreviations: DR, diabetic retinopathy; PBA, prevent blindness america; DM, diabetes mellitus; WHO, world health organization; DNA, deoxy ribonucleic acid; PSC, posterior subcapsular cataract; AR, aldose reductase; ARI, aldose reductase inhibitor

\section{Introduction}

Cataract is defined as opacity within the clear natural crystalline lens of the eye, which gradually results in vision deterioration. The World Health Organization (WHO) estimated that in 1990, out of the 38 million blind people worldwide, cataract accounted for $41.8 \%$ -almost 16 million people. ${ }^{1}$ With a projected increase in the geriatric population, WHO has estimated that there will be 54 million blind people aged 60years or older by the year 2020. ${ }^{1}$ Accordingly, cataract surgery will continue to weigh heavily on health care budgets in the developed nations. In the United States, cataract-related expenditure is estimated to be over \$3.4billion annually. ${ }^{2}$ In the developing world, the number of new cataract cases supersedes the rate of surgical removal. In Africa alone, only about $10 \%$ of the 500,000 new cases of cataract blindness each year are likely to have their sight restored surgically. It is estimated that if onset of cataract could be delayed by 10years, the annual number of cataract surgeries performed would be reduced by almost a half. ${ }^{2,3}$ This calls to question the risk factors of this multifactorial disease, which have been a litany of genetic, environmental, socioeconomic, and biochemical factors working in an interlaced fashion. The purpose of this paper is to provide an overview of the pathophysiology of cataract and the major interventions that have been deployed to help retard its progression.

\section{Pathogenesis of cataract}

The lens is composed of specialized proteins (called crystallins), whose optical properties are dependent on the fine arrangement of their three-dimensional structure and hydration. Membrane protein channels maintain osmotic and ionic balance across the lens, while the lens cytoskeleton provides for the specific shape of the lens cells, especially the fibre cells of the nucleus Protein-bound sulfhydryl (SH)-groups of the crystallins are protected against oxidation and cross-linking by high concentrations of reduced glutathione -'mother of all antioxidants'. Their molecular compositions, as well as tertiary and quaternary structures provide a high spatial and timely stability (heat-shock proteins) principally of the larger crystallins, which are able to absorb radiation energy (shortwave visible light, ultraviolet and infrared radiation) over longer time periods without basically changing their optical qualities. This provides substantial protective function also for the activity of various enzymes of the carbohydrate metabolism.

However, as ageing takes place, oxidative stress occurs which reflects an imbalance between the systemic manifestation of reactive oxygen species and a biological system's ability to readily detoxify the reactive intermediates or to repair the resulting damage. Disturbances in the normal redox state of cells can cause toxic effects through the production of peroxides and free radicals that damage all components of the cell, including proteins, lipids, and DNA. ${ }^{4}$ It is extensively recognized that oxidative stress is a significant factor in the genesis of senile cataract (the commonest cataract type), both in experimental animals. ${ }^{5}$ and in cultured lens models. ${ }^{6}$ The oxidative processes upsurge with age in the human lens, and concentration of proteins found is significantly higher in opaque lenses. ${ }^{7}$ This leads to break down and aggregation of protein, and culminates in damage to fiber cell membranes. ${ }^{8}$ Advanced that in the ageing eye, barriers develop that prevent glutathione and other protective antioxidants from reaching the nucleus in the lens, thus making it susceptible to oxidation.

In addition, ageing generally reduces the metabolic efficiency of the lens thus increasing its predisposition to noxious factors. Ageing provides the grounds where 'cataract noxae' can act and interact to induce the formation of a variety of cataracts, many of which are associated with high protein-related light scattering and discoloration. 
Resulting from ageing, the glucose metabolic pathway functions rather an aerobically with low energetic efficiency making protein synthesis, transport and membrane synthesis problematic. In addition, the syncytial metabolic function of the denucleated fiber cells has to be maintained by the epithelium and the small group of fiber cells, which still have their metabolic armamentarium. This results in a steep inside-out metabolic gradient, which is complicated by the fact that the lens behaves like an overhaul system, shutting off damaged groups of fiber cells -leading to wedge or sectorial cataract formation. All epithelial cells of the lens are subjected to light and radiation stress leading to alterations of the genetic code. Because defective cells cannot be extruded, these are either degraded (by apoptosis or necrosis), or they are moved to the posterior capsular area, where they contribute to the formation of posterior sub capsular cataracts (PSC).

The enzyme aldose reductase catalyzes the reduction of glucose to sorbitol through the polyol pathway, a process linked to the development of diabetic cataract. Extensive research has focused on the principal role of the AR pathway as the catalytic factor in diabetic cataract formation. It has been shown that the intracellular accumulation of sorbitol leads to osmotic changes resulting in hydropic lens fibers that degenerate and form sugar cataracts. ${ }^{9}$ In the lens, sorbitol is produced at a rapid rate than it is converted to fructose by the enzyme sorbitol dehydrogenase. In addition, the polar character of sorbitol prevents its intracellular removal through diffusion. The increased accumulation of sorbitol creates a hyperosmotic effect that results in an inflow of fluid to annul the osmotic gradient. Animal studies have shown that AR-mediated intracellular accumulation of polyols leads to a collapse and liquefaction of lens fibers, which ultimately results in the formation of lens opacities. ${ }^{10,11}$ These findings have led to the "Osmotic Hypothesis" of sugar cataract formation. Oxidative stress and osmotic imbalance can also result from nutritional and trace metals deficiencies, smoking, toxic substances including drugs abuses, alcohol etc., radiation (ultraviolet, electromagnetic waves etc). Leading to cataract formation. The exact pathophysiology of the above risk factors are however, clearly not understood.

\section{Retarding cataract progression: major interventions}

Since free radicals are principally implicated in cataract formation, major interventions in retarding cataract progression are targeted at annulling oxidation. Aldose reductase inhibitors (ARI) comprise a variety of structurally different compounds like plant extracts, animal tissues or specific small molecules. In diabetic rats, plant flavonoids such as quercetin (found in fruits) or the isoflavone genistein (found in clover soya, etc) have delayed diabetic cataract formation. ${ }^{12,13}$ Examples of natural products with known AR inhibitory activity are extracts from indigenous plants like Ocimum sanctum, Withania somnifera, Curcuma longa, and Azadirachtaindica. ${ }^{14,15}$ Several experimental studies support the role of ARI such as Ranirestat, ${ }^{16}$ Fidarestat. ${ }^{17}$ and Kinostat. ${ }^{18}$ in preventing and not only delaying diabetic cataract formation in diabetic rat and dog models. A number of different antioxidants have been reported to delay cataract formation and progression in diabetic animals. These include vitamin $\mathrm{C}$ and alpha lipoic acid, ${ }^{19}$ synergistic combination of vitamin $\mathrm{E}$ and insulin. ${ }^{20}$ endogenous pyruvate, ${ }^{21}$ carotenoid lutein-rich foods such as spinach and broccoli. ${ }^{6}$

Curcumin (in turmeric spice) has been well established as an anticataract agent. ${ }^{22,23}$ but the question of its bioavailability still remains unanswered. In addition, N-acetylcarnosine (NAC) eye drops have been proven to be effective free radical scavengers in improving visual function in cataract patients. ${ }^{24,25}$ This naturally occurring compound is believed to deacetylate and the resulting compound acts as an antioxidant and offers protection against glycation. ${ }^{26}$

\section{Discussion}

Since cataract is a major cause of avoidable blindness in the developing countries, the key to the success of Vision 2020: The right to sight initiative is a novel effort to wrestle cataract blindness by finding out the cause. While effective surgical procedures are available for treatment, the problem of post-operative complications, cost of surgery, and high number of people requiring surgery pose a considerable economic burden. It has been estimated that delaying cataract onset by 10years could reduce the need for surgery by as much as half. ${ }^{2,3}$ The respective causes of different type of cataracts must be known in order to understand the pathophysiology of the disease and its management.

Age-related or senile cataracts are mostly developed due to increase in oxidative stress in lens, ${ }^{4}$ either resulting from various systemic diseases such diabetes mellitus or imbalance in pro and anti-oxidants in the body, particularly the eyes. The deficiencies of some micronutrients also affect the antioxidant systems in the lenses of the eye. ${ }^{27,28}$ Many drug abuses as well as various toxins may cause oxidative damage and interrupt the lens growth as they bind to sulfhydryl groups, including glutathione peroxidase and $\mathrm{Na}^{+}$ $\mathrm{K}^{+}$ATPase, along with super oxide dismutase and catalase, which are responsible for the maintenance of clarity of the lens during oxidative stress. ${ }^{29}$ Radiation or electromagnetic waves can galvanize the exfoliation process in lens that leads to disruption in protein arrangement and oxidative systems. ${ }^{30}$

Elimination of the causes of cataract may reverse the cataractous changes at the earlier stages. Nutritional supplements and balancing antioxidants during old age and malnutrition have been reported in preventing senile cataract. ${ }^{31}$ A plethora of studies have reported that antioxidants (Vit E, Vit C, thiamine, riboflavin, lutein, flavonoids, carotenoids etc) ${ }^{6,32}$ can effectively prevent and remedy UVB-induced protein oxidation and photo-peroxidation of lipids in lens. The above notwithstanding, the mechanisms underpinning the development of cataract are still not down to earth. The potent interventions in managing this condition can only be arrived at, if the pathophysiology becomes very comprehensible and coherent.

\section{Conclusion}

The present study has reviewed the current understanding of the pathophysiology of cataract and the major interventions that have been employed to decelerate its progression. Despite the multifactorial etiology of cataract, the disease's pathogenesis is largely interlaced, with oxidative stress and free radical formation being central to cataract development. Ageing, diabetes and other metabolic and inherited defects, nutritional inadequacy, smoking, drug abuse (typically of steroids), toxins (classically of trace elements such as zinc and copper) and ultraviolet radiation, inter alia have been implicated as significant risk factors in the development of cataract. Antioxidants and aldose reductase inhibitors have been the mainstay of interventions that have been explored in the management of cataract progression. The need for further studies on cataract pathogenesis and management cannot be overemphasized, as there are lots of gray areas within the domain of this sight threatening condition. 


\section{Acknowledgments}

None.

\section{Conflicts of interest}

The authors declare that there is no conflict of interest.

\section{Funding}

None.

\section{References}

1. Thylefors B, Negrel AD, Pararajasegaram R, et al. Global data on blindness. Bull World Health Organ. 1995;73(1):115-121.

2. West SK, Valmadrid CT. Epidemiology of risk factors for age related cataract. Surv Ophthalmol. 1995;39(4):323-334.

3. Livingston PM, Carson CA, Taylor HR. The epidemiology of cataract: a review of the literature. Ophthalmic Epidemiol. 1995;2(3):151-164.

4. Lou MF. Redox regulation in the lens. Prog Retin Eye Res. 2003;22(5):657-682.

5. Truscott RJ. Age-related nuclear cataract-oxidation is the key. Exp Eye Res. 2005;80(5):709-725.

6. Gupta SK, Trivedi D, Srivastava S, et al. Lycopene attenuates oxidative stress induced experimental cataract development: An in vitro and in vivo study. Nutrition. 2003;19(9):794-799.

7. Boscia F, Grattagliano I, Vendemiale G, et al. Protein oxidation and lens opacity in humans. Invest Ophthalmol Vis Sci. 2000;41(9):2461-2465.

8. Harvey S, David Z. New York: Time Health Guide; [Updated and reviewed on 2010 June 23, Last accessed on 03.01.17], Cataract-Risk factors. 2000 .

9. Kinoshita JH. Mechanisms initiating cataract formation. Proctor lecture Invest Ophthalmol. 1974;13(10):713-724.

10. Kinoshita JH, Fukushi S, Kador P, et al. Aldose reductase in diabetic complications of the eye. Metabolism. 1979;28(4Suppl1):462-469.

11. Kador PF, Kinoshita JH. Diabetic and galactosaemic cataracts. Ciba Found Symp. 1984;106:110-131.

12. Varma SD, Devamanoharan PS, Morris SM. Prevention of cataracts by nutritional and metabolic antioxidants. Crit Rev Food Sci Nutrition. 1995;35(1-2):111-129.

13. Huang R, Shi F, Lei T, et al. Effect of the isoflavone genistein against galactose-induced cataracts in rats. Exp Biol Med (Maywood). 2007;232(1):18-125.

14. Halder N, Joshi S, Gupta SK. Lens aldose reductase inhibiting potential of some indigenous plants. $J$ Ethnopharmacol. 2003;86(1):113-116.

15. Moghaddam MS, Kumar PA, Reddy GB, et al. Effect of Diabecon on sugar-induced lens opacity in organ culture: mechanism of action. $J$ Ethnopharmacol. 2005;97(2):397-403.

16. Matsumoto T, Ono Y, Kuromiya A, et al. Long-term treatment with ranirestat (AS-3201), a potent aldose reductase inhibitor, suppresses diabetic neuropathy and cataract formation in rats. $J$ Pharmacol Sci. 2008;107(3):340-348.
17. Drel VR, Pacher P, Ali TK, et al. Aldose reductase inhibitor fidarestat counteracts diabetes-associated cataract formation, retinal oxidativenitrosative stress, glial activation, and apoptosis. Int $\mathrm{J} \mathrm{Mol} \mathrm{Med}$. 2008;21(6):667-676.

18. Kador PF, Betts D, Wyman M, et al. Effects of topical administration of an aldose reductase inhibitor on cataract formation in dogs fed a diet high in galactose. Am J Vet Res. 2006;67(10):1783-1787.

19. Kojima M, Sun L, Hata I, et al. Efficacy of $\alpha$-lipoic acid against diabetic cataract in rat. Jpn J Ophthalmol. 2007;51(1):10-13.

20. Yoshida M, Kimura H, Kyuki K, et al. Combined effect of vitamin E and insulin on cataracts of diabetic rats fed a high cholesterol diet. Biol Pharm Bull. 2004;27(3):338-344.

21. Varma SD, Hegde KR, Kovtun S. Attenuation and delay of diabetic cataracts by antioxidants: effectiveness of pyruvate after onset of cataract. Ophthalmologica. 2005;219(5):309-315.

22. Liao JH, Huang YS, Lin YC, et al. Anticataractogenesis mechanisms of curcumin and a comparison of its degradation products: an in vitro study. J Agric Food Chem. 2016;64(10):2080-2086.

23. Suryanarayana P, Saraswat M, Mrudula T, et al. Curcumin and turmeric delay streptozotocin-induced diabetic cataract in rats. 2005;46(6):2092-2099.

24. Babizhayev MA, Deyev AI, Yermakova VN, et al. Efficacy of $\mathrm{N}$-acetylcarnosine in the treatment of cataracts. Drugs $R D$. 2002;3(2):87-103.

25. Williams DL, Munday P. The effect of a topical antioxidant formulation including N-acetyl carnosine on canine cataract: a preliminary study. Vet Ophthalmol. 2006;9(5):311-316.

26. Boldyrev AA, Dupin AM, Bunin AYa, et al. The antioxidant properties of carnosine, a natural histidine-containing dipeptide. Biochem Int. 1987;15(6):1105-1113.

27. Bhat KS. Nutrition and cataract. Bulletin Nutr Fond India. 1993;14:1-3.

28. Shearer TR, David LL, Anderson RS, et al. Review of selenite cataract. Curr Eye Res. 1992;11(4):357-369.

29. Jobling AI, Augusteyn RC. What causes steroid cataracts? A review of steroid-induced posterior subcapsular cataracts. Clin Exp Optom. 2002;85(2):61-75.

30. Delcourt C, Carriere I, Ponton Sanchez A, et al. Light exposure and the risk of cortical, nuclear and posterior subcapsular cataracts: Pathologies Oculaires Liees a I'Age (POLA) study. Arch Ophthalmol. 2000;118(3):385-392.

31. Head KA. Natural therapies for ocular disorders. Part two: Cataract and Glaucoma. Altern Med Rev. 2001;6(2):141-166.

32. Wang J, Lofgren S, Dong X, et al. Dose-response relationship for $\alpha-$ tocopherol prevention of ultraviolet radiation induced cataract in rat. Exp Eye Res. 2011;93(1):91-97. 